\title{
RX J0123.4-7321, a Be/X-ray binary in the wing of the Small Magellanic Cloud
}

\author{
R. Sturm ${ }^{1}$, F. Haberl ${ }^{1}$, W. Pietsch ${ }^{1}$, and A. Udalski ${ }^{2}$ \\ ${ }^{1}$ Max-Planck-Institut für extraterrestrische Physik, Giessenbachstraße, 85748 Garching, Germany \\ e-mail: rsturm@mpe.mpg.de \\ 2 Warsaw Universy Observatory, Aleje Ujazdowskie 4, 00-478 Warsaw, Poland
}

Received 8 November 2012 / Accepted 13 January 2013

ABSTRACT

\begin{abstract}
Aims. To confirm faint Be/X-ray binary candidates from the XMM-Newton survey of the Small Magellanic Cloud, we searched for X-ray outbursts in archival ROSAT observations. We found that RX J0123.4-7321 was much brighter when detected with ROSAT than seen 16 years later by XMM-Newton.

Methods. We analysed the ROSAT observations and the OGLE I-band light curve of the optical counterpart to investigate the nature of the system.

Results. High long-term variability in the X-ray flux of a factor of $\sim 150$ was found between the ROSAT and XMM-Newton detections, indicating strong outburst activity during the ROSAT observations. The $I$-band light curve reveals long-term variability and regular outbursts with a period of $(119.9 \pm 2.5)$ days, indicating the orbital period of the binary system.

Conclusions. The large X-ray flux variations and the properties of the optical counterpart confirm RX J0123.4-7321 as a new Be/X-ray binary in the wing of the Small Magellanic Cloud.
\end{abstract}

Key words. Magellanic Clouds - galaxies: stellar content - stars: emission-line, $\mathrm{Be}$ - stars: neutron - X-rays: binaries

\section{Introduction}

Be/X-ray binaries (BeXRBs, e.g. Reig 2011) are binary systems composed of a compact object and a Be star of spectral type O9-B5 and luminosity class III-V. Be stars eject matter in the equatorial plane, which leads to the build-up of a decretion disc (Okazaki 2001; Ziolkowski 2002) around the star that produces emission lines and a near-infrared (NIR) excess. Both components show variability due to disc instabilities and sometimes due to interaction with the compact object. The compact object is in most cases a neutron star (NS). Systems with black holes are not known up to now. However, systems may contain white dwarfs (Li et al. 2012; Sturm et al. 2012a) that are observable at SMC distance as super-soft X-ray sources in the case of thermonuclear surface burning on the white dwarf. Caused by a kick experienced by the NS during the supernova explosion, the NS can have an eccentric orbit around the Be star. Accretion is possible when the NS is close to the decretion disc of the Be star during periastron passage. This causes so-called type-I outbursts that last for some days. Longer (several weeks) type-II outbursts are likely connected with decretion disc instabilities (Ziolkowski 2002).

The Small Magellanic Cloud (SMC) hosts a large population of BeXRBs. A literature search (e.g. Haberl \& Pietsch 2004; Coe et al. 2005; Liu et al. 2005; Laycock et al. 2010) reveals about 60 pulsars in more than 100 BeXRB systems (including candidates), which allows a comparison of their statistical properties with those of the Galactic sample. Most of the BeXRBs in the SMC are found in the bar where increased star formation $40 \mathrm{Myr}$ ago created them in large number (Antoniou et al. 2010). However, in the eastern wing of the SMC only a few systems are known, probably because this is a younger stellar population ( 10 Myr, Harris \& Zaritsky 2004). The BeXRBs in the wing might also be younger, as e.g. SXP 1062 suggests, which was found in a supernova remnant (Hénault-Brunet et al. 2012;
Haberl et al. 2012b). This might cause different properties of the wing sample. A literature search reveals three known BeXRB pulsars in the wing area with $P_{\text {spin }}<40$ s (Clark et al. 1997; Chakrabarty et al. 1998; Corbet et al. 2003) compared to only one (SXP 1062) pulsar with $P_{\text {spin }}>40 \mathrm{~s}$. In contrast to that, Knigge et al. (2011) found a bimodal spin distribution for the whole SMC with more systems above $P_{\text {spin }}>40 \mathrm{~s}$, which is likely caused by a bimodal distribution of orbital periods. The search for additional systems in the wing of the SMC is essential to enable a statistical comparison of the wing and bar population and exclude any observational bias.

The XMM-Newton survey of the SMC (Haberl et al. 2012a) revealed new BeXRBs in X-ray-bright state (Townsend et al. 2011; Sturm et al. 2011; Coe et al. 2011, 2012). In addition, several candidates for BeXRBs were found in the XMM-Newton SMC point-source catalogue (Sturm et al. 2013). One of these candidates is RX J0123.4-7321 (Haberl et al. 2000), which was previously detected with ROSAT (Trümper 1982) at much higher X-ray luminosity, but was not recognised as a BeXRB. In this paper, we present our analysis of the ROSAT observations and the OGLE (Udalski et al. 2008) data of the optical counterpart, which allow us to confirm RX J0123.4-7321 as a new BeXRB in the wing of the SMC with a determined orbital period.

\section{Analyses and results of X-ray observations}

RX J0123.4-7321 was detected four times in X-ray observations, as listed in Table 1 . The source was found in three ROSAT observations of SMCX-1 performed between October 1991 and June 1993 at an off-axis angle of $\sim 27.7^{\prime}$. The ROSAT position from Haberl et al. (2000, their source 460) is RA $(\mathrm{J} 2000)=$ $01^{\mathrm{h}} 23^{\mathrm{m}} 27^{\mathrm{s}} .2$ and $\operatorname{Dec}(\mathrm{J} 2000)=-73^{\circ} 21^{\prime} 25^{\prime \prime}$ with a $90 \%$ uncertainty of 4.6". This is consistent with the XMM-Newton position of the SMC-survey catalogue (Sturm et al. 2013, source 3003) 
Table 1. X-ray observations of RX J0123.4-7321.

\begin{tabular}{|c|c|c|c|c|c|c|c|c|c|c|}
\hline $\begin{array}{l}\text { Satellite } \\
\text { instrument }\end{array}$ & ObsID & $\mathrm{MJD}^{a}$ & $\begin{array}{c}\operatorname{Dur}^{b} \\
\text { (d) }\end{array}$ & $\begin{array}{l}\text { Orbit }^{c} \\
\text { Phase }\end{array}$ & $\begin{array}{l}\text { Exp } \\
(\mathrm{ks})\end{array}$ & Cts & $\begin{array}{c}F_{\text {observed }} \\
\left(\mathrm{erg} \mathrm{cm}^{-2} \mathrm{~s}^{-1}\right) \\
0.1-2.4 \mathrm{keV}\end{array}$ & $\begin{array}{c}F_{\text {model }} \\
\left(\mathrm{erg} \mathrm{cm}^{-2} \mathrm{~s}^{-1}\right) \\
0.2-4.5 \mathrm{keV}\end{array}$ & $\begin{array}{c}L^{d} \\
\left(\mathrm{erg} \mathrm{s}^{-1}\right) \\
0.1-2.4 \mathrm{keV}\end{array}$ & $\begin{array}{c}L^{d} \\
\left(\mathrm{erg} \mathrm{s}^{-1}\right) \\
0.2-4.5 \mathrm{keV}\end{array}$ \\
\hline ROSAT/PSPC & $\mathrm{rp} 400022$ & 48 & 0.96 & 0.65 & 16.6 & 936 & $8.84 \times 10^{-13}$ & $1.96 \times 10^{-12}$ & $6.43 \times 10^{35}$ & $1.07 \times 10^{36}$ \\
\hline ROSAT/PSPC & rp400022a01 & 48895.7 & 1.87 & 0.66 & 9.0 & 584 & $1.02 \times 10^{-12}$ & $2.27 \times 10^{-12}$ & $7.43 \times 10^{35}$ & $1.24 \times 10^{36}$ \\
\hline ROSAT/PSPC & rp400022a02 & 49141.0 & 1.20 & 0.70 & 11.9 & 147 & $1.95 \times 10^{-13}$ & $4.32 \times 10^{-13}$ & $1.42 \times 10^{35}$ & $2.36 \times 10^{35}$ \\
\hline XMM/EPIC & 0601212401 & 55011.6 & 0.41 & 0.71 & 25.4 & 58 & - & $1.55 \times 10^{-14}$ & - & $7.86 \times 10^{33}$ \\
\hline
\end{tabular}

Notes. ${ }^{(a)}$ Modified Julian date of the beginning of the observation. ${ }^{(b)}$ Duration of the observation. ${ }^{(c)}$ Phase $=0.5$ corresponds to the maximum of the optical outbursts. ${ }^{(d)}$ Unabsorbed luminosity. A source distance of $60 \mathrm{kpc}$ is assumed.

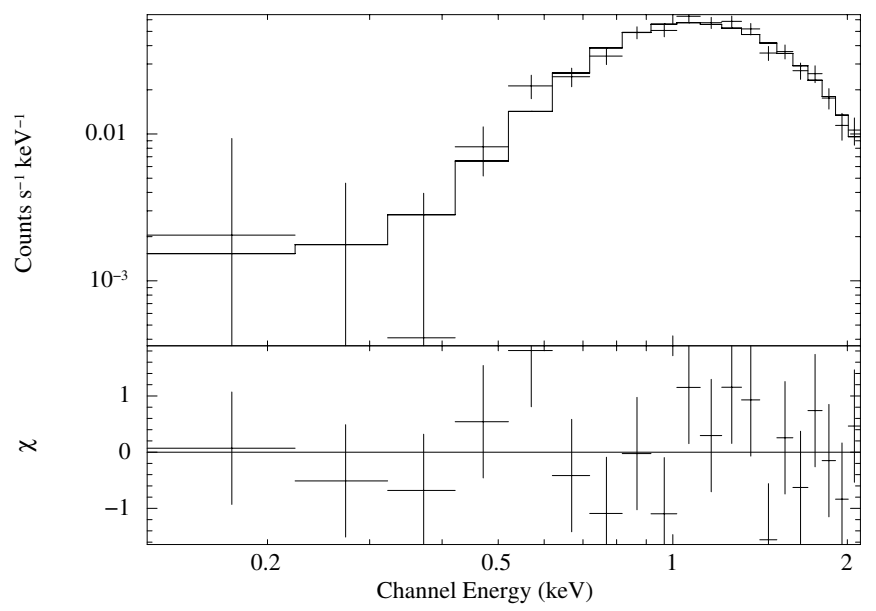

Fig. 1. Merged ROSAT/PSPC spectrum of RX J0123.4-7321 from observations rp400022n00 and rp400022a01 together with the best-fit power-law model. The lower panel gives the residuals in units of $\sigma$. of RA $(\mathrm{J} 2000)=01^{\mathrm{h}} 23^{\mathrm{m}} 27^{\mathrm{s}} .46$ and $\operatorname{Dec}(\mathrm{J} 2000)=-73^{\circ} 21^{\prime} 23^{\prime \prime} .4$ with a $1 \sigma$ uncertainty of $1.1^{\prime \prime}$.

ROSAT spectra were extracted using the ftools (Blackburn 1995) task xselect. For spectral analysis, we merged observations rp400022n00 and rp400022a01 to increase the statistics and used xspec (Arnaud 1996) version 12.7.0u to fit the spectrum with a power-law model and photoelectric absorption by a Galactic column density set to $N_{\mathrm{H} \text {,gal }}=3.3 \times 10^{20} \mathrm{~cm}^{-2}$ (a foreground H I map was kindly provided by Erik Muller, see also Muller et al. 2003) and an additional column density, accounting for the interstellar medium of the SMC and source-intrinsic absorption. We find a good description of the data by this model with $\chi^{2} /$ d.o.f. $=14.8 / 17$. For the SMC and source-intrinsic absorption we obtain $N_{\mathrm{H}, \mathrm{smc}}=4.32_{-0.28}^{+0.36} \times 10^{21} \mathrm{~cm}^{-2}$. The total lineof-sight $N_{\mathrm{H}, \mathrm{smc}}$ column density at the position of RX J0123.47321 is $3.02 \times 10^{21} \mathrm{~cm}^{-2}$ (Stanimirovic et al. 1999), pointing to some intrinsic absorption in the binary system. The photon index is determined to be $\Gamma=1.18_{-0.54}^{+0.63}$, which suggests a neutron-star $\operatorname{BeXRB}(\Gamma \approx 1$, Haberl \& Pietsch 2004), but we cannot exclude an active galactic nucleus $(\mathrm{AGN}, \Gamma \approx 1.75$, Tozzi et al. 2006). The ROSAT spectrum together with the best-fit model is presented in Fig. 1.

The XMM-Newton detection is based on only 58 counts. However, hardness ratios are consistent with a BeXRB nature of the source. Assuming the same spectral shape for the ROSAT and XMM-Newton detections and comparing their fluxes, we find a high long-term variability of a factor of $146 \pm 20$, which would be atypical for an AGN.

We used a Bayesian odds ratio (Gregory \& Loredo 1996; Haberl et al. 2008) and a Rayleigh $Z_{1}^{2}$ (Buccheri et al. 1983; Haberl \& Zavlin 2002) test to search for the spin period of

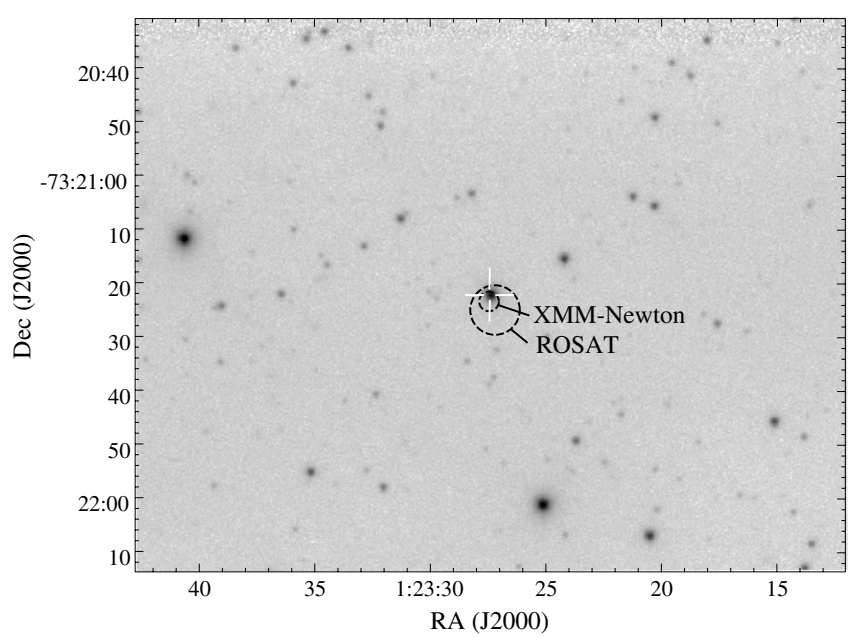

Fig. 2. OGLE III $I$-band finding chart of RX J0123.4-7321, marked with a white cross. Circles indicate the $90 \%$ position uncertainties of the ROSAT and XMM-Newton detections.

the NS. The search for pulsations in the ROSAT data with best statistics (rp400022n00) in the (1-200) s band did not reveal convincing periodicities. Note that the ROSAT observation was performed with individual exposures between 200 and $1300 \mathrm{~s}$, preventing the detection of long periods. The best candidate for the spin period with $1 \sigma$ uncertainty is found at $17.34529(55) \mathrm{s}$. Since this period has a significance of $3.3 \sigma$ and could not be confirmed in the other observations, probably owing to the lower number of detected counts, the spin period needs further confirmation.

\section{Analyses and results of optical observations}

An I-band finding chart from OGLE III is presented in Fig. 2. An optical counterpart, GSC 09142-02001, is found with an angular separation to the XMM-Newton position of $1.3^{\prime \prime}$ for the entry in the photometric catalogue of Massey (2002), giving $U=14.48 \mathrm{mag}, B=15.39 \mathrm{mag}, V=15.45 \mathrm{mag}$, and $R=15.42$ mag as measured on MJD 51 186. The NIR counterpart, 2MASS 01232743-7321223 (Skrutskie et al. 2006), is found with an angular separation of $1.1^{\prime \prime}$ with $J=15.32 \mathrm{mag}$, $H=14.82 \mathrm{mag}$, and $K_{\mathrm{S}}=15.06 \mathrm{mag}$ measured on MJD 51034 . The corresponding OGLE sources are OGLE III SMC121.2.50 and OGLE IV SMC733.26.24 with a separation of $1.2^{\prime \prime}$ to the X-ray source. The OGLE $I$-band value of $I \approx 15.4 \mathrm{mag}$ is a typical magnitude of the variable light curve (see below). The magnitudes and colours are characteristic of a B star in the SMC. From optical spectroscopy, Evans et al. (2004) classified the source (2dFS 3624) as a B1-3 III star. Since the density of 


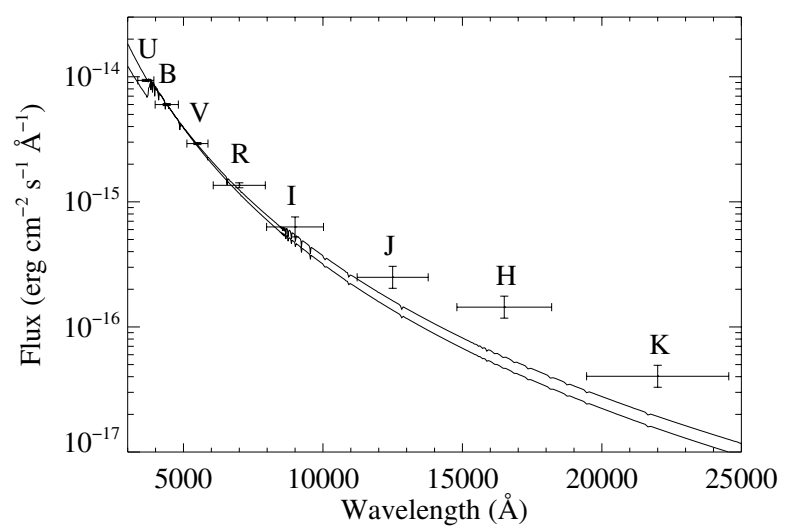

Fig. 3. Optical and NIR photometry of RX J0123.4-7321 dereddened and compared with normalised Kurucz model atmospheres for a B1 III and a B3 III star. Labels give filters.

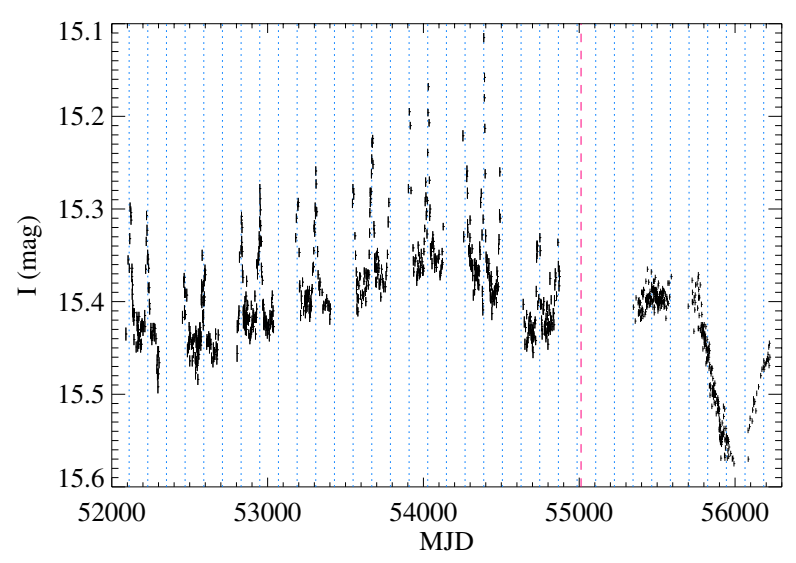

Fig. 4. Optical $I$-band light curve of RX J0123.4-7321 from OGLE III (before MJD 55 000) and OGLE IV (after MJD 55 000). Predicted outburst maxima are indicated by the dotted blue lines. The time of the $X M M$-Newton observation is marked by the dashed red line.

early-type stars is relatively low in the wing of the SMC, a correlation with the X-ray source by chance is unlikely.

We dereddened these magnitudes to correct for a Galactic foreground of $E_{B-V}=0.07 \mathrm{mag}$ (Schwering \& Israel 1991) and compared them to a Kurucz model atmosphere (Kurucz 1979; Howarth 2011) of a B1 III and a B3 III star $\left(T_{\text {eff }}=\right.$ $25000,18000 \mathrm{~K}$ and $\log (g)=3.0)$ in Fig. 3. The models have been normalised to the $B$-band measurement. To account for possible variability of the source and the non-simultaneous observations, we included an uncertainty of 0.2 mag for the $I$ and NIR magnitudes. There is evidence for an NIR excess, likely caused by the circum-stellar decretion disc, which supports the Be classification of the star.

The long-term I-band light curve from OGLE phases III and IV is presented in Fig. 4. In addition to a long-term variability, regular outbursts are seen. The light curve is typical for Be stars. A Lomb-Scargle periodogram (Lomb 1976; Scargle 1982) of the OGLE III data confirms the variability with a period of 119.87 days (Fig. 5). We derive an ephemeris for the $I$-band outburst maximum of

$\mathrm{MJD}=54387.14 \pm 0.10+(119.9 \pm 2.5) \times N$,

with $N$ an integer. The folded $I$-band light curve is presented in Fig. 6. The average outburst duration (FWHM) is 30 days, as derived from the folded light curve. However, the evolution can be different for individual outbursts. For several outbursts we find a double-peaked shape, as also indicated in the folded light curve.

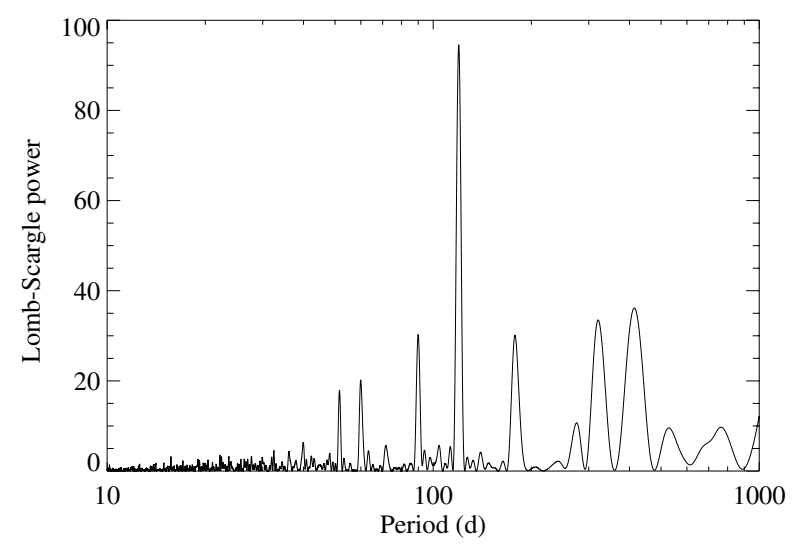

Fig. 5. Lomb-Scargle periodogram of the I-band light curve of RX J0123.4-7321 from OGLE III data before MJD 55000.

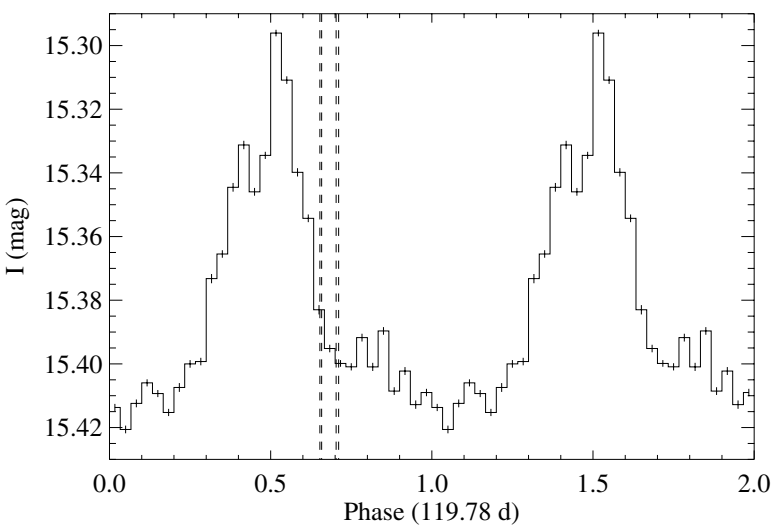

Fig. 6. OGLE III light curve of RX J0123.4-7321 for MJD $<55000$ folded with the $119.87 \mathrm{~d}$ period. Phase $=0.5$ corresponds to MJD 54387.14. The phases of the dates of the X-ray observations are indicated by dashed lines (see Table 1).

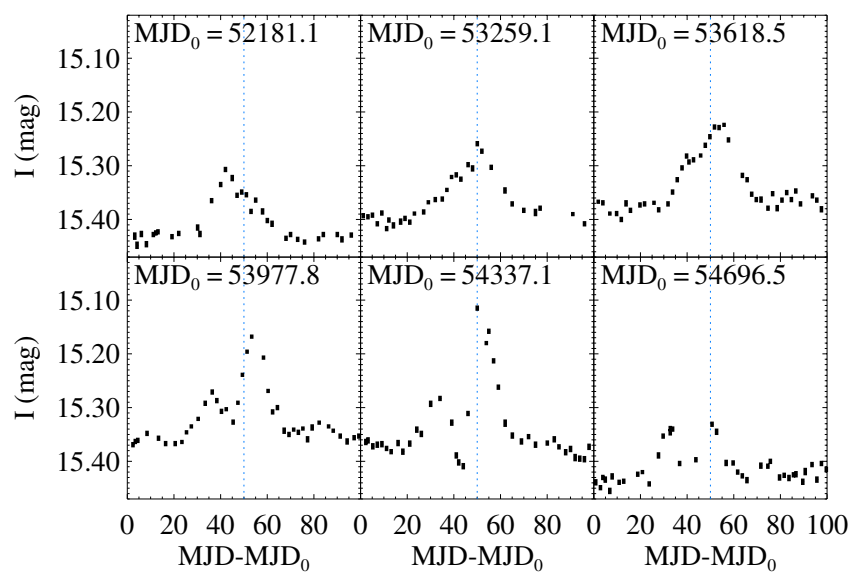

Fig. 7. OGLE III light curves of RX J0123.4-7321 for six individual outbursts. Predicted outburst maxima are indicated by the dotted blue lines.

Other outbursts only show one peak, where the maximum can be $\sim 15$ days before or after the expected time of the outburst maximum. Examples for individual outbursts are presented in Fig. 7. In other BeXRBs, typically a fast rise and exponential decay is observed during optical outbursts (Bird et al. 2012). In general, individual outburst profiles will depend on NS-orbit parameters and perturbations of the decretion disc (Okazaki 1991).

All X-ray observations were made shortly after the times of possible optical outbursts, as indicated by the vertical lines in Fig. 6. If both optical and X-ray outburst appear during periastron passage, we would expect quasi-simultaneous outbursts 
A\&A 551, A96 (2013)

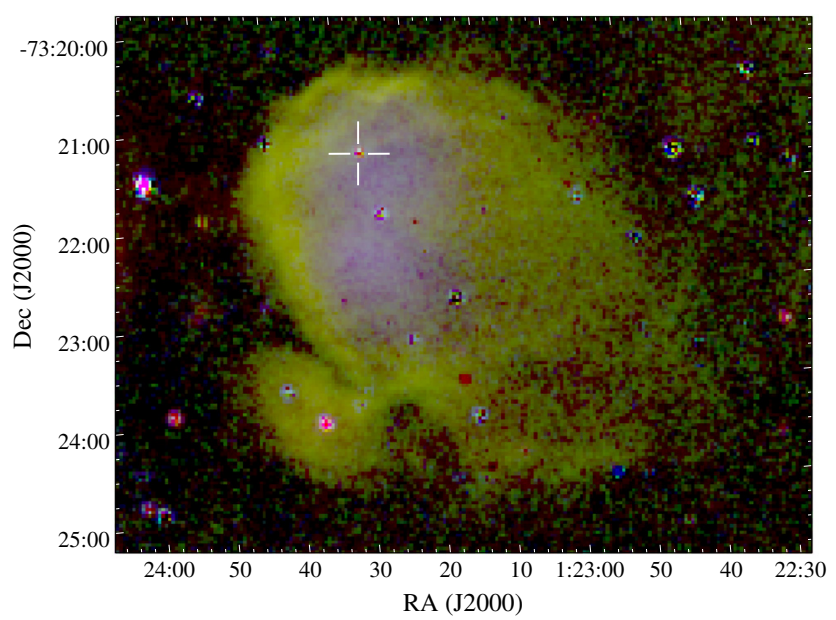

Fig. 8. Continuum-subtracted emission-line image of DEM S 160 from MCELS (Winkler et al. 2005). Red/green/blue give logarithmically scaled intensities for $H \alpha /[\mathrm{S} \mathrm{II}] /[\mathrm{O} \mathrm{III}]$. RX J0123.4-7321 is marked with a white cross.

as e.g. observed in AX J0058-720 (Haberl \& Pietsch 2007) and IGR J05414-6858 (Sturm et al. 2012b). However, we note that the X-ray observations have been performed during times without OGLE coverage where the occurrence of optical outbursts is uncertain. The recently observed decrease in the $I$-band light curve and the vanishing of the outbursts can be caused by a loss of the decretion disc around the Be star. Interestingly, first the outbursts were missing after MJD 55400, although the quiescent $I$-band flux was at a similar level when outbursts have been observed around MJD 53 000. Between MJD 55700 and 56000 , a drop of the $I$-band flux was observed, followed by a brightening, probably due to a recovering of the decretion disc.

We note that RXJ0123.4-7321 is along the line of sight to the H II region DEM S 160 (Davies et al. 1976), see Fig. 8. However, due to the high angular separation of $\sim 1^{\prime}$ to the centre of DEM S 160, which corresponds to a distance of at least $17.5 \mathrm{pc}$, both sources are likely not directly connected to each other, but may have formed during the same star-formation event.

\section{Discussion and conclusions}

We analysed ROSAT and OGLE data of RXJ0123.4-7321. Due to the high X-ray variability by a factor of at least $\sim 150$ and the properties of the optical counterpart, we identify RX J0123.4-7321 as a new BeXRB in the wing of the SMC. The $I$-band light curve of the optical counterpart shows a typical behaviour for a Be star in a BeXRB. The periodic outbursts are likely caused by binarity and reveal the orbit period of the NS of $P_{\text {orb }}=119.78 \mathrm{~d}$. This places RX J0123.4-7321 in the sample of long-orbit systems (Knigge et al. 2011). From the Corbet relation (Corbet 1984; Corbet et al. 2009), we can roughly estimate the spin period of the neutron star to be between 10 and $800 \mathrm{~s}$. Therefore, the source is unlikely to be identified with the $2.16 \mathrm{~s}$ pulsar XTE J0119-731 (Corbet et al. 2003) that was found by RXTE with an angular separation of $18^{\prime}$. No indication of this period was found in the X-ray periodograms of RX J0123.4-7321. More X-ray observations of the system in outburst are necessary, to confirm the possible spin period of $17.3 \mathrm{~s}$ and the neutron-star nature of the compact object. If the current low-state of the $I$-band emission is caused by a loss of the decretion disc, no X-ray activity is expected. Follow-up X-ray observations are most likely to be successful during new I-band outbursts, which may indicate interaction of the NS and the decretion disc. Optical outbursts could reappear with brightening $I$-band emission, and if so, will be found in future OGLE monitoring. The system is included in the X-Ray variables OGLE Monitoring (XROM, Udalski 2008) system.

Acknowledgements. The OGLE project has received funding from the European Research Council under the European Community's Seventh Framework Programme (FP7/2007-2013) / ERC grant agreement no. 246678 to AU. R.S. acknowledges support from the BMWI/DLR grant FKZ 50 OR 0907.

\section{References}

Antoniou, V., Zezas, A., Hatzidimitriou, D., \& Kalogera, V. 2010, ApJ, 716, L140

Arnaud, K. A. 1996, in Astronomical Data Analysis Software and Systems V, eds. G. H. Jacoby, \& J. Barnes, ASP Conf. Ser., 101, 17

Bird, A. J., Coe, M. J., McBride, V. A., \& Udalski, A. 2012, MNRAS, 423, 3663 Blackburn, J. K. 1995, in Astronomical Data Analysis Software and Systems IV, eds. R. A. Shaw, H. E. Payne, \& J. J. E. Hayes, ASP Conf. Ser., 77, 367

Buccheri, R., Bennett, K., Bignami, G. F., et al. 1983, A\&A, 128, 245

Chakrabarty, D., Levine, A. M., Clark, G. W., \& Takeshima, T. 1998, IAU Circ., 7048, 1

Clark, G. W., Remillard, R. A., \& Woo, J. W. 1997, ApJ, 474, L111

Coe, M. J., Edge, W. R. T., Galache, J. L., \& McBride, V. A. 2005, MNRAS, 356,502

Coe, M. J., Haberl, F., Sturm, R., et al. 2011, MNRAS, 414, 3281

Coe, M. J., Haberl, F., Sturm, R., et al. 2012, MNRAS, 424, 282

Corbet, R., Markwardt, C. B., Marshall, F. E., et al. 2003, IAU Circ., 8064, 4

Corbet, R. H. D. 1984, A\&A, 141, 91

Corbet, R. H. D., Coe, M. J., McGowan, K. E., et al. 2009, in IAU Symp. 256, eds. J. T. van Loon, \& J. M. Oliveira, 361

Davies, R. D., Elliott, K. H., \& Meaburn, J. 1976, MmRAS, 81, 89

Evans, C. J., Howarth, I. D., Irwin, M. J., Burnley, A. W., \& Harries, T. J. 2004, MNRAS, 353, 601

Gregory, P. C., \& Loredo, T. J. 1996, ApJ, 473, 1059

Haberl, F., \& Pietsch, W. 2004, A\&A, 414, 667

Haberl, F., \& Pietsch, W. 2007, A\&A, 476, 317

Haberl, F., \& Zavlin, V. E. 2002, A\&A, 391, 571

Haberl, F., Filipović, M. D., Pietsch, W., \& Kahabka, P. 2000, A\&AS, 142, 41

Haberl, F., Eger, P., \& Pietsch, W. 2008, A\&A, 489, 327

Haberl, F., Sturm, R., Ballet, J., et al. 2012a, A\&A, 545, A128

Haberl, F., Sturm, R., Filipović, M. D., Pietsch, W., \& Crawford, E. J. 2012b, A\&A, 537, L1

Harris, J., \& Zaritsky, D. 2004, AJ, 127, 1531

Hénault-Brunet, V., Oskinova, L. M., Guerrero, M. A., et al. 2012, MNRAS, 420, L13

Howarth, I. D. 2011, MNRAS, 413, 1515

Knigge, C., Coe, M. J., \& Podsiadlowski, P. 2011, Nature, 479, 372

Kurucz, R. L. 1979, ApJS, 40, 1

Laycock, S., Zezas, A., Hong, J., Drake, J. J., \& Antoniou, V. 2010, ApJ, 716, 1217

Li, K. L., Kong, A. K. H., Charles, P. A., et al. 2012, ApJ, 761, 99

Liu, Q. Z., van Paradijs, J., \& van den Heuvel, E. P. J. 2005, A\&A, 442, 1135

Lomb, N. R. 1976, Ap\&SS, 39, 447

Massey, P. 2002, ApJS, 141, 81

Muller, E., Staveley-Smith, L., Zealey, W., \& Stanimirović, S. 2003, MNRAS, 339,105

Okazaki, A. T. 1991, PASJ, 43, 75

Okazaki, A. T. 2001, PASJ, 53, 119

Reig, P. 2011, Ap\&SS, 332, 1

Scargle, J. D. 1982, ApJ, 263, 835

Schwering, P. B. W., \& Israel, F. P. 1991, A\&A, 246, 231

Skrutskie, M. F., Cutri, R. M., Stiening, R., et al. 2006, AJ, 131, 1163

Stanimirovic, S., Staveley-Smith, L., Dickey, J. M., Sault, R. J., \& Snowden, S. L. 1999, MNRAS, 302, 417

Sturm, R., Haberl, F., Coe, M. J., et al. 2011, A\&A, 527, A131

Sturm, R., Haberl, F., Pietsch, W., et al. 2012a, A\&A, 537, A76

Sturm, R., Haberl, F., Rau, A., et al. 2012b, A\&A, 542, A109

Sturm, R., Haberl, F., Pietsch, W., et al. 2013, A\&A, submitted

Townsend, L. J., Coe, M. J., Corbet, R. H. D., et al. 2011, MNRAS, 410, 1813

Tozzi, P., Gilli, R., Mainieri, V., et al. 2006, A\&A, 451, 457

Trümper, J. 1982, Adv. Space Res., 2, 241

Udalski, A. 2008, Acta Astron., 58, 187

Udalski, A., Szymanski, M. K., Soszynski, I., \& Poleski, R. 2008, Acta Astron., 58,69

Winkler, P. F., Young, A. L., Braziunas, D., et al. 2005, in BAAS, Am. Astron. Soc. Meet. Abstracts, 37, 132.03

Ziolkowski, J. 2002, Mem. Soc. Astron. It., 73, 1038 